\title{
A GENERIC PROPERTY OF THE BOUNDED SYZYGY SOLUTIONS
}

\author{
FLORIN N. DIACU
}

(Communicated by George G. Papanicolaou)

\begin{abstract}
For a set of masses having positive measure, excepting eventually a negligible set of initial conditions, every noncollinear bounded solution of the planar three-body problem that has a syzygy configuration encounters an infinity of such configurations. Along a noncollinear syzygy solution, the set of syzygy configuration instants is discrete.
\end{abstract}

\section{INTRODUCTION}

Very little is known about the syzygy solutions of the three-body problem although these special configurations present interest in applications for the conjunction-opposition and eclipses phenomena in the solar system. We have proved in a previous paper [D] that, in the planar case, the set of initial conditions leading to syzygy solutions is nonempty and open. Sitnikov [Si] and later Moser [M] have studied oscillatory motion in connection with stability problems and found out that, in the spatial restricted isosceles three-body problem, there exist unbounded solutions having an infinity of syzygy configurations. Samarov [Sa] proved that, along a solution of the general isosceles three-body problem with nonnegative constant of energy (i.e., when usually at least a particle escapes from the system), there is only a finite number of syzygy configurations.

The goal of this note is to give a generic property of the noncollinear bounded syzygy solutions of the planar three-body problem. We prove that there exists a set of masses having positive measure such that if a bounded noncollinear solution of the eqs. $(*)$ below has a syzygy configuration then it has an infinity of such configurations, excepting eventually a set of solutions of Lebesgue measure zero. This is mainly a consequence of a result due to Arnol'd [A] concerning quasi-periodic solutions, the above-mentioned result of the author [D] and the classical ergodic theorem of Poincaré. Finally we observe that, along a noncollinear solution, the sequence of syzygy configuration instants cannot accumulate in finite time.

Received by the editors May 15, 1990 and, in revised form, April 13, 1991.

1991 Mathematics Subject Classification. Primary 70F10, 70F15; Secondary 34C35, 58F11, $28 \mathrm{~A} 75$.

Key words and phrases. 3-body problem, syzygy solution, ergodic theory, Lebesgue measure. 


\section{EQUATIONS OF MOTION AND DEFINITIONS}

Consider the equations

$$
\begin{cases}\dot{\mathbf{q}}_{i}=m_{i}^{-1} \mathbf{p}_{i}, & i=1,2,3, \\ \dot{\mathbf{p}}_{i}=\partial_{i} U(\mathbf{q}), & i=1,2,3,\end{cases}
$$

describing the motion in the planar three-body problem, where $\mathbf{q}_{i}=\left(q_{i}^{1}, q_{i}^{2}\right)$, $\mathbf{p}_{i}=m_{i} \dot{\mathbf{q}}_{i}, i=1,2,3$, are the position vectors and momenta, the constants $m_{i}>0, i=1,2,3$, are the masses, $\partial_{i}$ is the $i$ th gradient, and

$$
U: \mathbb{R}^{6} \backslash \Delta \rightarrow \mathbb{R}_{+}, \quad U(\mathbf{q})=\sum_{1 \leq i<j \leq 3} m_{i} m_{j} /\left|\mathbf{q}_{i}-\mathbf{q}_{j}\right|,
$$

represents the potential function of the system of particles,

$$
\Delta=\bigcup_{1 \leq i<j \leq 3}\left\{\mathbf{q}=\left(\mathbf{q}_{1}, \mathbf{q}_{2}, \mathbf{q}_{3}\right) ; \mathbf{q}_{i}=\mathbf{q}_{j}\right\}
$$

being the collision set and $|\cdot|$ denoting the Euclidean norm in $\mathbb{R}^{2}$.

Standard results of the theory of differential equations ensure, for given initial conditions $(\mathbf{q}, \mathbf{p}) \in\left(\mathbb{R}^{6} \backslash \Delta\right) \times \mathbb{R}^{6}$, the existence and uniqueness of an analytic solution $(\mathbf{q}, \mathbf{p})$ of the eqs. $(*)$, defined on a maximal interval $\left(t^{-}, t^{+}\right)$, $-\infty \leq t^{-}<t^{+} \leq+\infty$. It is known that if $t^{-}$or $t^{+}$is finite then the solution experiences a singularity due to a double or a triple collision at the corresponding time moment [P]. It means that if an orbit of the planar three-body problem does not begin or end in a collision, then it is defined on the whole $\mathbb{R}$.

Without loss of generality we may consider the eqs. $(*)$ restricted to the invariant set $\mathbf{Q} \times \mathbf{P}$, where

$$
\mathbf{Q}=\left\{\mathbf{q} ; m_{1} \mathbf{q}_{1}+m_{2} \mathbf{q}_{2}+m_{3} \mathbf{q}_{3}=0\right\}, \quad \mathbf{P}=\left\{\mathbf{p} ; \mathbf{p}_{1}+\mathbf{p}_{2}+\mathbf{p}_{3}=0\right\},
$$

which physically means that the motion is considered relative to the center of mass of the three-body system.

Observe that the masses define the open set

$$
\mathbb{R}_{+}^{3}=\left\{\left(m_{1}, m_{2}, m_{3}\right) ; m_{i}>0, i=1,2,3\right\}
$$

in $\mathbb{R}^{3}$. To different choices of the masses correspond different problems, i.e., different forms of the eqs. $(*)$.

Definition 1. A solution ( $(\mathbf{q}, \mathbf{p})$ of the eqs. $(*)$ is called collinear if for every $t \in\left(t^{-}, t^{+}\right)$there exists a straight line (depending on $t$ ) that contains the bodies at this time. In case the line is independent on $t$ then the solution is called rectilinear.

Definition 2. A solution (q, $\mathbf{p})$ of the eqs. $(*)$ is said to have a syzygy configuration at time $t_{0} \in\left(t^{-}, t^{+}\right)$if there is a straight line in the plane containing all the bodies at $t_{0}$ instant. A solution is called syzygy in case it encounters at least one syzygy configuration. Observe that since $t_{0}$ cannot be $t^{-}$or $t^{+}$, collisions are not syzygy configurations. 


\section{THE GENERIC PROPERTY}

Denote by $\mathbf{S}$ the set of initial conditions in $(\mathbf{Q} \backslash \Delta) \times \mathbf{P}$ leading to bounded noncollinear syzygy solutions of the eqs. $(*)$. Let $\mathbf{C}_{r}(0), r \in \mathbb{N}$, be the hypersphere of dimension 11 and radius $r$ contained in the phase space and centered in the origin of the frame. Denote by $\mathbf{B}_{r}$, the set of the initial data of solutions that do not leave the 12-dimensional ball bounded by $\mathbf{C}_{r}(0)$, and let $\mathbf{B}:=\bigcup_{r \in \mathbb{N}} \mathbf{B}_{r}$. Then, obviously, $\mathbf{S} \subset \mathbf{B}$. Denote $\mathbf{S}_{r}=\mathbf{S} \cap \mathbf{B}_{r}$. We will prove the following result.

Theorem. There exists a set of masses of positive Lebesgue measure in $\mathbb{R}_{+}^{3}$ such that for any choice of the masses in this set the corresponding form of the eqs. (*) has the following property: excepting a set of Lebesgue measure zero included in $\mathbf{S}$, every $(\mathbf{q}, \mathbf{p})(0) \in \mathbf{S}$ leads to a solution $(\mathbf{q}, \mathbf{p})$, of the eqs. $(*)$, having an infinity of syzygy configurations. The set of syzygy-configuration instants along any solution is formed by isolated points.

Proof. First observe that since the set of initial conditions leading to collisions is of Lebesgue measure zero [S], almost all solutions are defined on $\mathbb{R}$. We further need a result due to Arnol'd [A], which can be stated in the following way, suitable for our purposes. For the planar three-body problem there is a class of masses of positive Lebesgue measure (namely, when one mass is large and the others are small) and a set $\mathbf{G}$ of initial conditions such that every $(\mathbf{q}, \mathbf{p})(0) \in \mathbf{G}$ leads to a quasi-periodic solution of the eqs. $(*)$ (for their definition see, e.g., [SM]). Moreover $\mathbf{G}$ has the structure of a Cantor set with positive Lebesgue measure. By the definition of quasi-periodic solutions it is easy to show that the orbits determined by $\mathbf{G}$ do not encounter collisions and are bounded.

On the other side we have proved in [D] that the set $\Sigma$ of initial conditions in $(\mathbf{Q} \backslash \Delta) \times \mathbf{P}$ leading to noncollinear syzygy solutions of the eqs. $(*)$ is nonempty and open. In [D] we gave a general example of syzygy solutions that can be used in order to see that $\mathbf{G} \cap \Sigma \neq \varnothing$; more precisely, we can always find initial data in $\mathbf{G}$ determining a syzygy solution. Since $\mathbf{G}$ is a Cantor set, $\Sigma$ is open and $\mathbf{G} \cap \Sigma \subset \mathbf{S}$, and it follows that $\mathbf{S}$ contains a Cantor set of the same type and has, consequently, positive Lebesgue measure in the phase space.

Since the set of solutions bounded by $\mathbf{C}_{r}(0)$, defined above, is of finite measure, invariant for the eqs. $(*)$ and

$$
\operatorname{div}\left(m_{1}^{-1} \mathbf{p}_{1}, m_{2}^{-1} \mathbf{p}_{2}, m_{3}^{-1} \mathbf{p}_{3}, \partial_{1} U(\mathbf{q}), \partial_{2} U(\mathbf{q}), \partial_{3} U(\mathbf{q})\right)=0,
$$

by the Recurrence Theorem of Poincaré, for every $(\mathbf{q}, \mathbf{p})(0) \in \mathbf{B}_{r}$, excepting eventually a negligible set $\mathbf{M}_{r}$ of initial conditions, we have: for every $\varepsilon>0$ and $t^{*} \in \mathbb{R}$, there exists an increasing sequence $\left(t_{n}\right)_{n \in \mathbb{N}}, t_{n}=t_{n}\left(t^{*}, \varepsilon\right)$, with $\lim _{n \rightarrow \infty} t_{n}=\infty$, such that $\left\|(\mathbf{q}, \mathbf{p})\left(t_{n}\right)-(\mathbf{q}, \mathbf{p})\left(t^{*}\right)\right\|<\varepsilon, n \in \mathbb{N}$.

Using the above-described structure of $\mathbf{S}$ we may draw the conclusion that for some $(\mathbf{q}, \mathbf{p})(0) \in \mathbf{S}$ there exists a neighborhood of $\mathbf{S}$ of diameter $\delta>0$ having positive Lebesgue measure. Without loss of generality we can suppose that $(\mathbf{q}, \mathbf{p})$ has a syzygy configuration at $t_{0} \neq 0$ and choose $t^{*}=0$ and $\varepsilon=\delta$. Thus, $(\mathbf{q}, \mathbf{p})\left(t_{0}\right)$ is near a syzygy configuration and may be considered as the initial value of another solution, let us say as a point $\left(\mathbf{q}^{*}, \mathbf{p}^{*}\right)(0)$ of the phase space. Since the distance between $\left(\mathbf{q}^{*}, \mathbf{p}^{*}\right)(0)$ is less than $\delta$, by the continuity of the solution with respect to initial data, it follows that $\left(\mathbf{q}^{*}, \mathbf{p}^{*}\right)(0) \in \mathbf{S}$, i.e., $(\mathbf{q}, \mathbf{p})(0)$ leads to a sygyzy configuration at some instant $t_{0}^{*}$ near $t_{0}$, but not 
near 0 . Repeating this process for $t_{1}, t_{2}, \ldots, t_{n}, \ldots$, by choosing suitable values of $t^{*}$ and the same $\varepsilon=\delta$, we obtain an increasing sequence of time moments $\left(t_{n}^{*}\right)_{n \in \mathbb{N}}$ with $t_{n}^{*}$ near $t_{n}$ for every $n$, such that $(\mathbf{q}, \mathbf{p})$ encounters syzygy configurations at each $t_{n}^{*}$.

Thus we have seen that for any $r \in \mathbb{N}$, every $(\mathbf{q}, \mathbf{p}) \in \mathbf{S}_{r} \backslash \mathbf{M}_{r}$ has an infinity of syzygy configurations. Observe that since $\mathbf{M}_{r}$ has Lebesgue measure zero, the set $\mathbf{M}=\bigcup_{r \in \mathbb{N}} \mathbf{M}_{r}$ is also negligible. Now, since $\mathbf{S} \backslash \mathbf{M} \subset \mathbf{B}=\bigcup_{r \in \mathbb{N}} \mathbf{B}_{r}$ and $\mathbf{B}_{r} \subset \mathbf{B}_{r+1}, r \in \mathbb{N}$, it follows that for any $(\mathbf{q}, \mathbf{p}) \in \mathbf{S} \backslash \mathbf{M}$ there exists an index $r \in \mathbb{N}$ such that $(\mathbf{q}, \mathbf{p}) \in \mathbf{S}_{r} \backslash \mathbf{M}_{r}$, i.e., $(\mathbf{q}, \mathbf{p})$ has the required property. The conclusion concerning the infinity of sygyzy configurations, therefore follows, for almost all bounded noncollinear syzygy solutions.

The last part of the theorem is obvious by the fact proved in [D] (which uses the identity theorem of analytic functions) that if the set of syzygy configuration moments of a solution of the $N$-body problem, $N \geq 3$, has a finite accumulation point, then the solution is collinear.

\section{ACKNOWLEDGMENTS}

I am indebted to Bernold Fiedler for valuable comments concerning a previous form of this note.

\section{REFERENCES}

[A] V. I. Arnol'd, The classical theory of perturbations and the problem of stability of planetary systems, Soviet Math. Dokl. 3 (1962), 1008-1012.

[D] F. N. Diacu, On the planar Syzygy solutions of the 3-body problem, Celestial Mech. 46 (1989), 119-128.

[M] J. K. Moser, Stable and random motions in dynamical systems, Princeton Univ. Press, Princeton, NJ, 1971.

[P] P. Painlevé, Leçons sur la théorie analytiques des equations différentielles, Hermann, Paris, 1897.

[S] D. G. Saari, Imporbability of collisions in newtonian gravitational systems, Trans. Amer. Math. Soc. 162 (1971), 267-271; 168 (1972), 521; 181 (1973), 351-368.

[SA] K. L. Samarov, Regularization of the isosceles three-body problem for a nonzero area vector, Soviet Math. Dokl. 18 (1977), 245-249.

[SM] C. L. Siegel and J. K. Moser, Lectures on celestial mechanics, Springer-Verlag, Berlin, Heidelberg, and New York, 1971.

[Si] K. A. Sitnikov, The existence of oscillary motion in the three-body problem, Soviet Phys. Dokl. 5 (1961), 647-650.

Centre de Recherches Mathématiques, Université de Montréal, CP 6128-A, Montréal, QuÉBEC H3C 3J7, CANADA

Current address: Department of Mathematics and Statistics, University of Victoria, P. O. Box 3045, Victoria, British Columbia, V8W 3P4 Canada

E-mail address: diacu@sol.uvic.ca 